\title{
From Telomere Crisis via Dicentric Chromosomes to Kataegis and Chromothripsis
}

All linear chromosomes are capped at their ends, the telomeres, by special telomeric repeat sequences [Riethman, 2008]. Since these sequences cannot be replicated by the regular DNA replication machinery, a specialized telomerase reverse transcriptase (TERT) takes care of maintaining their length at the end of each round of DNA replication [Autexier and Lue, 2006]. Loss of TERT activity may cause a few rare autosomal recessive, dominant and $\mathrm{X}$-linked syndromes, such as dyskeratosis congenita 1, idiopathic pulmonary fibrosis and myelodysplastic syndrome, and may be involved in ageing [Armanios and Blackburn, 2013; Vermeij et al., 2015]. Single chromosomes undergo, upon loss of their telomeric repeat sequences, breakage-fusion-bridge cycles, producing dicentric chromosomes, which may subsequently be degraded into mitotically stable dicentric supernumerary marker chromosomes [Murnane, 2012; Spittel et al., 2014; Poot and Haaf, 2015]. Products of breakage-fusionbridge cycles are rarely observed, since cell cycle checkpoints prevent their proliferation. In contrast, cells with persistent telomere dysfunction and deficient in p53 action experience a prolonged G2 phase due to ATM/ATRand Chk1/Chk2-mediated inhibition of Cdk1/CyclinB. They then bypass mitosis, enter a second $\mathrm{S}$ phase, which results in whole-genome reduplication, and become tetraploid [Davoli et al., 2010]. Upon restoration of telomere protection, these tetraploid cells resume cell division cycles and proliferate further. (c) 2016 S. Karger AG, Base

$1661-8769 / 16 / 0066-0259 \$ 39.50 / 0$
Recently, an equally dramatic effect of telomere damage has been demonstrated. Maciejowski et al. [2015] generated a derivative of a human TERT expressing retinal pigment in an epithelial cell line (RPE-1) and disabled the $\mathrm{Rb}$ and $\mathrm{p} 53$ pathways. Subsequently, they induced telomere crises, by expressing a dominant negative allele of TRF2, which deprotects telomeres and induces telomere fusions [van Steensel et al., 1998]. As expected, 53BP1-containing foci indicative of telomere dysfunction emerged, ATM kinase signaling was activated and cell proliferation impaired. While metaphases with telomere fusions were observed, no micronuclei were seen. The dicentric chromosomes persisted through anaphase to become long chromatin bridges that induced rupture of the nuclear envelopes. The chromatin bridges were coated by replication protein A. Subsequently, the cytoplasmic $3^{\prime} \rightarrow 5^{\prime}$ exonuclease TREX1 converted the chromatin bridges into single-stranded DNA (ssDNA), which facilitated their resolution. Thereupon, the authors isolated cell clones and confirmed in clones with aneuploidy and/ or marker chromosomes by telomere fusion PCR that telomere fusion had indeed taken place. By whole-genome sequencing clusters of genomic rearrangements, affecting one or more chromosomes and exhibiting hallmarks of chromothripsis, such as close spatial clustering, random fragment orientation and oscillating copy number states were found [Korbel and Campbell, 2013; Poot and Haaf, 2015]. Those were in particular terminal dele- 
tions, which is consistent with products of telomere fusion. Also more proximal chromothripsis-associated changes being limited to a single chromosome arm were found. In no case an entire chromosome was affected.

Since the chromatin bridges acquire replication protein A, they have to contain extended streches of ssDNA, one of the target substrates of APOBEC enzymes [Roberts et al., 2012]. This class of cytosine deaminases converts clusters of cytosine to thymidine or uracil in long stretches of ssDNA formed in the vicinity of double-strand breaks and stalled DNA replication forks [Roberts et al., 2012]. This process is known as kataegis. Maciejowski et al. [2015] found 29 clusters of point mutations in 7 out of 10 cell clones, which were absent from the parental cell lines. These clusters exhibited the cardinal features of kataegis as observed earlier in human cancers. First, the clusters were often found within 1-2 kb of genomic breakpoints and were predominantly associated with chromothripsis rearrangements. Second, the clusters showed a pronounced preference for $\mathrm{C}>\mathrm{T}$ and $\mathrm{C}>\mathrm{G}$ mutations within a $\mathrm{TpC}$ context, which is the classic signature of APOBEC3A/B activity [Roberts and Gordenin, 2014]. Third, the mutation clusters were processive, which indicates that the damage occurred on a single strand of DNA.
The close association of this form of chromothripsis with kataegis and the finding that it involves chromatin bridges, but no micronucleus formation, clearly sets it apart from the previously discovered type of chromothripsis [Zhang et al., 2015]. Both studies have been performed in RPE-1 cell lines. In untransformed RPE-1 cells, nocodazole treatment and p53 knockdown was used to induce chromothripsis through micronucleus formation [Zhang et al., 2015]. For chromothripsis after telomere crisis to occur, both the $\mathrm{Rb}$ and the p53 pathways have to be disabled [Maciejowski et al., 2015]. This is unlikely to occur in the germline, but it may very well take place in somatic cells during malignant disease, where chromothripsis is frequently observed [Cai et al., 2014]. Therefore, the present study primarily provides novel insights into cancer, but inasmuch as it also links telomere damage with chromosomal breakage-fusion-bridge cycles and double-strand DNA breaks, it may also pertain to the formation of some types of complex chromosome rearrangements in the germline [Poot and Haaf, 2015]. The close association of chromothripsis events with kataegis may prompt further in-depth studies in both constitutional and cancer genetics.

Martin Poot

\section{References}

Armanios M, Blackburn EH: The telomere syndromes. Nat Rev Genet 13:693-704 (2012).

-Autexier C, Lue NF: The structure and function of telomerase reverse transcriptase. Annu Rev Biochem 75:493-517 (2006).

-Cai H, Kumar N, Bagheri HC, von Mering C, Robinson MD, Baudis M: Chromothripsislike patterns are recurring but heterogeneously distributed features in a survey of 22,347 cancer genome screens. BMC Genomics 15: $82(2014)$.

Davoli T, Denchi EL, de Lange T: Persistent telomere damage induces bypass of mitosis and tetraploidy. Cell 141:81-93 (2010).

Korbel JO, Campbell PJ: Criteria for inference of chromothripsis in cancer genomes. Cell 152:1226-1236 (2013).
Maciejowski J, Li Y, Bosco N, Campbell PJ, de Lange T: Chromothripsis and kataegis induced by telomere crisis. Cell 163:1641-1654 (2015).

Murnane JP: Telomere dysfunction and chromosome instability. Mutat Res 730:28-36 (2012)

Poot M, Haaf T: Mechanisms of origin, phenotypic effects and diagnostic implications of complex chromosome rearrangements. Mol Syndromol 6:109-133 (2015).

Riethman H: Human telomere structure and biology. Annu Rev Genomics Hum Genet 9:1-19 (2008).

Roberts SA, Gordenin DA: Hypermutation in human cancer genomes: footprints and mechanisms. Nat Rev Cancer 14:786-800 (2014).

Roberts SA, Sterling J, Thompson C, Harris S, Mav D, et al: Clustered mutations in yeast and in human cancers can arise from damaged long single-strand DNA regions. Mol Cell 46: 424-435 (2012).
Spittel H, Kubek F, Kreskowski K, Ziegler M, Klein E, et al: Mitotic stability of small supernumerary marker chromosomes: a study based on 93 immortalized cell lines. Cytogenet Genome Res 142:151-160 (2014).

van Steensel B, Smogorzewska A, de Lange T: TRF2 protects human telomeres from end-toend fusions. Cell 92:401-413 (1998).

-Vermeij WP, Hoeijmakers JH, Pothof J: Genome integrity in aging: human syndromes, mouse models, and therapeutic options. Annu Rev Pharmacol Toxicol 56:427-445 (2016).

Zhang CZ, Spektor A, Cornils H, Francis JM, Jackson EK, et al: Chromothripsis from DNA damage in micronuclei. Nature 522:179-184 (2015). 